\title{
WHO must prioritise the needs of older people in its response to the covid-19 pandemic
}

The last author of this letter by Peter G Lloyd-Sherlock et al (BMJ 2020;368:m1164, doi:10.1136/bmj.m1164) has asked to be removed from the article. Luis Miguel Gutierrez did not approve a revision made late during editing. His name has been removed from the article online with the agreement of all authors. 